\title{
A Proof Theory \\ for \\ Constructive Default Logic
}

\author{
Yao-Hua Tan
}

\author{
Erasmus University Rotterdam, Department of Computer Science, \\ P.O. box 1738, 3000 DR Rotterdam, The Netherlands, tan@cs.few.eur.nl \\ Free University Amsterdam, Department of Mathematics and Computer \\ Science, De Boelelaan 1081a, 1081 HV Amsterdam, The Netherlands, \\ tan@cs.vu.nl
}

\begin{abstract}
We present what we call Constructive Default Logic (CDL) - a default logic in which the fixedpoint definition of extensions is replaced by a constructive definition which yield so-called constructive extensions. Selection functions are used to represent explicitly the control of the reasoning process in this default logic. It is well-known that Reiter's original default logic lacks, in general, a default proof theory. We will show that CDL does have a default proof theory, and we will also show that this is related to the fact that $\mathrm{CDL}$ has the existence property for constructive extensions and that it also has the semi-monotonicity property. Furthermore, we will also show that, with respect to some counter-examples that were suggested by Lukaszewicz, constructive extensions yield more intuitive conclusions than Reiter's extensions. Hence, constructive default logic does not only have heuristic advantages over Reiter's default theory from a computational point of view, but it is also more adequate with respect to knowledge representation.
\end{abstract}

1 This research was partly supported by the ESPRIT III BRA Project 6156 DRUMS 2. 


\section{Introduction}

A variety of non-monotonic formalisms have been proposed to formalize defeasible reasoning. For a comprehensive survey of non-monotonic reasoning the reader is referred to [Bes89] and [Luk90]. It is well-known that defeasible reasoning is very complex from a computational viewpoint. For example, Kautz and Selman showed in [KS91] that even simple default theories in Reiter's default logic are very hard to compute.

One of the sources of the complexity problems in default logic is its non-constructive character. In [Rei80] Reiter defines extensions in default logic by a fixed-point definition, which is not constructive. This definition only allows one to check whether a set of sentences is an extension, but it provides no information about how to generate an extension. So, the actual reasoning process (and all the decisions how to solve conflicts!) that leads to an extension is left implicit in Reiter's default logic. In recent years it was observed by several researchers that the complexity problem of default logic can be heuristically improved by making the default reasoning process more constructive (for an extensive discussion of this point see e.g. [Moi92] and [FM92a,b]). We have developed a constructive version of Reiter's default logic. Constructive Default Logic $(C D L)$ is a default logic in which the fixed-point definition of extensions is replaced by a constructive definition. From a computational point of view constructive extensions have a heuristic advantage over Reiter extensions. A detailed discussion about the heuristic advantages of constructive default logic in comparison to Reiter's default logic can be found in [TT92].

Apart from being non-constructive it is also well-known that Reiter's original default logic lacks a default proof theory for non-normal default rules, which was already observed by Reiter himself to be a weakness of his logic. In this paper we will show that CDL does have a default proof theory, and we will also show that this is intrinsically related to the fact that default theories in CDL always have a constructive extension (the existence property), and that CDL also has the semi-monotonicity property. Reiter's default logic lacks these two properties. Furthermore, we will show that, with respect to some counter-examples that were suggested by Lukaszewicz, constructive extensions yield more intuitive conclusions than Reiter's extensions. Hence, constructive default logic does not only have heuristic advantages over Reiter's default theory from a computational point of view, but it is also more adequate with respect to knowledge representation.

\section{Constructive Default Logic}

Constructive default logic is default logic in which the normal fixed-point extensions of Reiter are replaced by so-called constructive extensions. Research on constructive default logic was reported earlier in [Tan91], [Tan92a], [Tan92b], [TT91a] and [TT92]. Constructive 
extensions are parameterized by a selection function that is able to express the choice for the extension to be generated. This selection function can be considered as a setting of a set of control parameters that guides the reasoning. Thus we can parameterize the branching of the defeasible reasoning process.

\subsection{Extensions in Reiter's Default Logic}

We give a brief summary of Reiter's default logic (see [Rei80], or [Bes89]). Default logic is a classical logic extended with extra defeasible inference rules, the so-called default rules. We write default rules as

$$
\left(: "{ }_{1}, \ldots, " \mathrm{n} / \bullet\right)
$$

where , " ${ }_{i}$ and - are logical formulas. The formula is called the prerequisite, ${ }_{i}$ the justifications and $\bullet$ the conclusion of the default rule. The intuitive interpretation of such a default rule is: if is believed, and all " $i$ are consistent with the set of beliefs, then one can infer the conclusion $\bullet$. A default rule is called normal if its justification is identical to its conclusion, i.e. if it is of the form ( : " / "). A default rule is called semi-normal if it is of the form ( : " •/・). A default theory $\Delta=\langle W, D\rangle$ consists of a set of logical formulas $W$ and a set of default rules $D$. The deductive closure of the union of $W$ and a set of conclusions of a default theory is called an extension E. In what follows $T h(S)$ denotes the deductive closure in a logic $L$ of a set $S$ of $L$-formulas, i.e. $\operatorname{Th}(S)=\left\{{ }^{\sim} \mid S f^{\sim}{ }^{u}\right\}$. To distinguish Reiter's extensions from our constructive extensions, we will call the first Rextensions.

\section{Definition 2.1}

A set of sentences $E$ is an R-extension of the default theory $\Delta=\langle W, D\rangle$, if $E \underset{\mathrm{i}=0}{=} E_{\mathrm{i}}$, where each layer $E_{\mathrm{i}}$ is defined as follows:

for $i=0$,

$$
E_{0}=W,
$$

and for $i \geq 0$,

$$
E_{\mathrm{i}+1}=\operatorname{Th}\left(E_{\mathrm{i}}\right) \text { fi }\left\{\bullet \mid\left(: "{ }_{1}, \ldots,{ }_{\mathrm{n}} / \bullet\right) £ D, \quad £ E_{\mathrm{i}} \text {, and } \quad{ }_{1}, \ldots, \quad{ }_{\mathrm{n}} \quad E\right\} .
$$

Note that this definition is a fixed-point definition, which in principle is not constructive. In the definition of the layer $E_{\mathrm{i}+1}$ it is required that the formulas " ${ }_{1}, \ldots$, "n are not contained in $E$. Hence, the definition of each layer $E_{\mathrm{i}+1}$ depends on the final outcome $E$. A simple example illustrates this definition. Suppose $\Delta=\langle W, D\rangle$ with $W=\{b\}$ and $D=$ (b : $\quad p / f$ ). This default rule says that if something is a bird, and it is not known to be a 
penguin, then one can assume it can fly. One can easily verify that the R-extension of $\Delta$ is $E=$ $T h(\{b, f\})$, hence the conclusion that it can fly, i.e. the formula $f$, is contained in $E$. However, if we have the information in $W$ that the object is a penguin, i.e. $W^{\prime}=\{b, p\}$, then the default theory $\Delta^{\prime}=\left\langle W^{\prime}, D\right\rangle$ has the R-extension $E^{\prime}=T h(\{b, p\})$, which does not contain the formula $f$. This implies that in default logic conclusions are not preserved under information growth.

\subsection{Selection Functions}

A selection function, which will be denoted by $\S$, selects a subset of default conclusions from the set of all default conclusions that can be derived at a certain layer. Indices $i$ from an index set $I$ are added to indicate that the selection is made at the $i$-th reasoning step. The general definition of a selection function is as follows.

\section{Definition 2.2.1}

Let $\boldsymbol{\square}$ be a logic and let $U$ be a set of well-formed formulas of $\boldsymbol{\square}$. Let $\mathbf{D}(\mathrm{U})$ denote the set of all subsets $V$ " $U$, and let $I$ be an index set. The function $\S:$ I x $\mathbf{\square}(\mathrm{U}) \mathbf{\square}(\mathrm{U})$ is called a selection function if for every subset $V$ of $U$ and every index $i ; \S(i, V)$ " $V$.

\section{Definition 2.2.2}

Let $D$ be a set of defaults. The set of consequences of $D$, denoted as Cons $(D)$ is defined by $\operatorname{Cons}(D)=\left\{\bullet \mid\left(:{ }^{\prime} 1, \ldots,{ }_{\mathrm{n}} / \bullet\right) £ D\right\}$. Suppose $\Delta=\langle W, D\rangle$ is a default theory and $\S: I \times \square(U) \square(U)$ is a selection function. We call $\S$ a selection function related to $\Delta$ if $U=$ $\operatorname{Cons}(D)$. The set of selection functions related to $\Delta$ is denoted by $\operatorname{Sel}(\Delta)$.

In this paper the index set $I$ will always be the set of natural numbers, with the usual ordering. The condition in Definition 2.2.1 says that $\$$ should not select a formula that is not contained in $V$. Instead of $\$(i, V)$ we will also write $\operatorname{simply} \S_{i}(V)$. We will say that a selection function is an identity selection function if for all $V " \operatorname{Cons}(D)$ and for all $i$ holds $\S_{\mathrm{i}}(V)=V$. And we will say that a selection is an empty selection function if for all $V$ " $C o n s(D)$ and for all $i$ holds $\S_{i}(V)={ }^{\circ}$.

\subsection{Constructive Extensions}

First we define the notion of a $\$$-constructive extension. This is a constructive extension of which the construction is controlled by a selection function $\S$. 


\section{Definition 2.3.1}

Let $\Delta=\langle W, D\rangle$ be a default theory and $\S$ be a selection function related to $\Delta$. A set of sentences $C^{\S}$ is called the $\$$-constructive extension of the default theory $\Delta$, if $C^{\S}=_{\mathrm{i}=0} C^{\S} \mathrm{i}$, where

$$
\begin{aligned}
& \text { for } i=0 \text {, } \\
& C^{\S_{0}}=W, \\
& C^{\S_{i+1}}=\operatorname{Th}\left(C^{\S_{i}}\right) \text { fi } \S_{i+1}\left(\operatorname{Cons}\left(D^{\S_{i+1}}\right)\right),
\end{aligned}
$$

where

$$
D_{\mathrm{i}+1}^{\S_{1}}=\left\{\left(:{ }_{1}, \ldots,{ }_{\mathrm{n}} / \bullet\right) £ D \mid £ C_{\mathrm{i}}^{\S_{1}} \text {, and } \quad{ }_{1}, \ldots, \quad{ }_{\mathrm{n}} \quad \operatorname{Th}\left(C_{\mathrm{1}}^{\S}\right)\right\} .
$$

We will call default logic based on \$-constructive extensions Constructive Default Logic $(C D L)$. In the sequel we will refer to Reiter's default logic with R-extensions as $D L$. If it is clear from the context, we will usually omit the $\$$ prefix, and simply call it constructive extension. For a given default theory $\Delta=\langle W, D\rangle$ there is a collection of such $\$$ constructive extensions, parameterized by selection functions $\S £ \operatorname{Sel}(\Delta)$.

\section{Definition 2.3.2}

Let $\Delta=\langle W, D\rangle$ be a default theory and suppose $\$$ is a selection function related to $\Delta$. A default rule $\left(: "{ }_{1}, \ldots,{ }_{n} / \bullet\right) £ D$ is called applicable at stage $i$ if $\left(:{ }_{1}, \ldots,{ }_{n} / \bullet\right) £$ $D \S_{\mathrm{i}}$. We say that a default $\left(:{ }^{\prime} 1, \ldots,{ }_{\mathrm{n}} / \bullet\right) £ D$ is used in principle by $\S$ at stage $i$ if it is applicable and $\bullet £ \$_{\mathrm{i}}\left(\operatorname{Cons}\left(D_{\mathrm{1}}\right)\right)$. In this case we also say that the formula $\bullet$ is selected by $\$$ at stage $i$.

The next example shows that the choice of a selection function is very important.

\section{Example 2.3.3}

Let $\Delta=\langle W, D\rangle$ be a default theory with

$$
W={ }^{\circ} \quad \text { and } \quad D=\{(: p / p),(: p / p)\}
$$

Consider the identity selection function $\$$, i.e. for all $i$ and $V$ " $\operatorname{Cons}(D)$ we have $\S_{i}(V)=V$, then $\$$ generates the constructive extension $\mathscr{C} h(\{p, p\})$, which is inconsistent. This is due to the fact that both defaults in $D$ can be applied and are selected by $\S$ at stage $C{ }_{1}$ in the construction of $C^{\S}$, i.e. $\S_{1}\left(\operatorname{Cons}\left(D^{\S}\right)\right)=\{p, \quad p\}$.

From this example follows immediately the following observation. 


\section{Observation 2.3 .4}

A constructive extension $C^{\S}$ of a default theory $\Delta=\langle W, D\rangle$ can be inconsistent, although $W$ is consistent.

This observation clearly distinguishes $C D L$ from $D L$, because Reiter proved that every Rextension of a default theory $\Delta=\langle W, D\rangle$ is inconsistent if and only if $W$ is inconsistent.

\section{Proposition 2.3.5 (see Corollary 2.2 in [Rei80])}

Let $E$ be an R-extension of the default theory $\Delta=\langle W, D\rangle$, then $E$ is inconsistent if and only if $W$ is inconsistent.

From Example 2.3.3 also follows immediately that $D L$ and $C D L$ are not equivalent for normal defaults.

\section{Observation 2.3.6}

If $\Delta=\langle W, D\rangle$ is a default theory of which $D$ consists only of normal default rules, then it is generally not the case that for every constructive extension $C^{\S}$ of $\Delta$ there is an R-extension $E$ of $\Delta$ such that $E=C^{\S}$.

This distinguishes $C D L$ from every alternative default logic proposed in the literature, because all the alternative default logics I know of are equivalent to $D L$ with respect to normal default rules (for an extensive survey of alternative default logics see [FM92a,b]).

At first sight the type of inconsistency in Example 2.3.1 might look like something that has to be prevented at all costs. However, in recent years more and more researchers have become aware that inconsistencies are simply a fact of life, and that we must be able to represent them in our knowledge representation formalisms. For example Gabbay and Hunter argued extensively for the importance of maintaining inconsistencies in common sense reasoning [GH91,92]. Actually there are already different logical formalisms that allow inconsistencies. For example Belnap's 4-valued logic [Bel77] and da Costa's paraconsistent logic [dC74]. In [Tan92b] I discuss the Bi-Modular System (BMS), a meta-level architecture for nonmonotonic reasoning, that is based on a 4-valued logic similar to Belnap's. Since BMS corresponds to $C D L$ in a well-defined way, this 4 -valued logic can also be used for $C D L$. The basic idea is that the deductive closure operator $(T h)$ in Definition 2.3.1 is replaced by a deductive closure operator under Belnap's logic. This topic of representing inconsistencies will be further investigated in another paper. Here we take a more conservative viewpoint, and discuss how we can impose constraints on the selection functions in order to prevent inconsistencies between default conclusions. 


\section{Definition 2.3.7 (Local Consistency Constraint)}

Let $\Delta=\langle W, D\rangle$ be a default theory, and let $\S$ be a selection function related to $\Delta$. We say that $\$$ satisfies the Local Consistency constraint, or short $L C$-constraint, if for all $i$ and every $V$ " $\operatorname{Cons}(D)$ we have that $\S_{\mathbf{i}}(V)$ is consistent.

This solves the inconsistency in Example 2.3.3, because the identity selection function $\$$ selects $\S_{1}\left(D^{\$_{0}}\right)=\{p, \quad p\}$, hence it does not satisfy the LC-constraint. Inconsistencies between default conclusions can also occur between default conclusions that are selected at different stages in the construction of a constructive extension. Hence, in addition to the local consistency constraint we also need a global consistency constraint.

\section{Definition 2.3.8 (Global Consistency Constraint)}

Let $\Delta=\langle W, D\rangle$ be a default theory, and let $\$$ be a selection function related to $\Delta$. We say that $\$$ satisfies the Global Consistency constraint, or short GC-constraint, if for every $V$ " $\operatorname{Cons}(D)$ we have that ${ }_{\mathrm{i}=0} \S_{\mathrm{i}}(V)$ is consistent.

In addition to inconsistent default conclusions there is another phenomenon, which we call non-groundedness, which is typical for constructive extensions. Consider the following example.

\section{Example 2.3.9 (Non-Groundedness example)}

Let $\Delta=\langle W, D\rangle$ be a default theory with

$$
W=\{q \quad p\} \quad \text { and } \quad D=\{(: p / q)\}
$$

Consider the identity selection function $\$$, i.e. for all $i$ and $V$ " $\operatorname{Cons}(D)$ we have $\S_{\mathrm{i}}(V)=V$, then $\S$ generates the constructive extension $=C \mathscr{C} h(\{q, p\})$. This is counterintuitive, because the justification $p$ of the default rule $(: p / q)$ is violated by the conclusion of the implication $q \quad p$.

What happens in this example is that the default rule defeats its own justification indirectly. First the default can be applied at a certain stage, but at a later stage the conclusion of this default leads to a violation of the justification of this default rule. Also this problem can be solved by imposing a constraint, the so-called non-self-defeating constraint, on selection functions.

\section{Definition 2.3.10}

A default $\left(:{ }^{\prime \prime} 1, \ldots,{ }^{\prime} \mathrm{n} / \bullet\right) £ D$ that in principle is used at stage $i$ by a selection function $\S$ is called defeated by $\$$, if there is some $j \geq i$ such that $\quad " \mathrm{k} £ T h(C \S)$ for some $k$ with $1 \leq k \leq \mathrm{n}$. We call $\$$ self-defeating if there is some stage $i$ and some formula $\bullet$ that is selected by $\S$ at $i$ such that all defaults $\left(:{ }^{1}{ }_{1}, \ldots,{ }_{n} / \bullet\right) £ D \$_{i}$ with consequence $\bullet$ are 
defeated by $\S$. If $\$$ is not self-defeating, then we say that $\$$ is non-self-defeating $(N S D)$, or that it satisfies the NSD-constraint, or simply that it is NSD.

From this definition it is clear that empty selection functions satisf trivially.

Observation 2.3 .11

Every empty selection function satisfies the NSD-constraint.

It turns out that the NSD-constraint is a very strong constraint. In part: the NSD-constraint subsumes the consistency constraints, where subsumptio: defined as follows.

\section{Definition 2.3.12 (Subsumption between constraints))}

Let $A$ and $B$ be two costraints on selection functions. We say that $A$ subsumes $B$, written $A \quad B$, if we have for every selection function $\$$ that

If $\S$ satisfies the $A$-constraint, then $\S$ satisfies the $B$-constraint.

Note that the subsumption relation is reflexive and transitive. The GC-constraint subsumes the LC-constraint, and the NSD-constraint subsumes the GC-constraint. Hence, we have the following subsumption relation between the NSD-, GC- and LC-constraints.

NSD $\quad$ GC $\quad$ LC

We only prove that the NSD-constraint subsumes the GC-constraint. The ot relation GC LC is obvious. Moreover, NSD LC follows immediately by the transitivity of the subsumption relation.

\section{Proposition 2.3.13 (NSD-constraint subsumes GC-constraint)}

For every selection function $\$$ holds;

If $\$$ satisfies the NSD-constraint, then $§$ satisfies the GC-constraint.

Proof.

Suppose $\$$ is a selection function related to $\Delta=\langle W, D\rangle$ that does not satisfy the GCconstraint, then there is a stage $i$ in the construction of the construcive extension $C^{\S}$ of $\Delta$ such that ${ }_{\mathrm{j}=0}^{\mathrm{i}} \S_{\mathrm{j}}\left(\operatorname{Cons}\left(D^{\S_{\mathrm{j}}}\right)\right)$ is inconsistent. Due to this inconsistency we know that $\operatorname{Th}\left(C{ }^{\S}\right)$ contains every formula, and so does $C^{\S_{i+1}}$. Hence, it is obvious that for every formula $\bullet$ that 
is selected by $\S$ at stage $i$ holds that all defaults $\left(:{ }_{1} 1, \ldots,{ }^{n} / \bullet\right) £ D_{1+1}$ are defeated by $\$$. This implies that $\$$ is self-defeating. $\quad$ fl

We also have the following result about the NSD-constraint.

\section{Proposition 2.3.14}

Let $C \S$ be a constructive extension of the default theory $\Delta=\langle W, D\rangle$ with $\S$ satisfying the NSD-constraint, then $C \S$ is inconsistent if and only if $W$ is inconsistent.

Proof. Straightforward.

It can happen that in the construction of stage $j$ it turns out that some default conclusions that were derivable at an earlier stage $i<j$ are no longer derivable at $j$. In other words, it is not guaranteed that if $i<j$, then $\S_{\mathrm{i}}\left(\operatorname{Cons}\left(D^{\$_{\mathrm{i}}}\right)\right)$ " $\S_{\mathrm{j}}\left(\operatorname{Cons}\left(D^{\S} \mathrm{j}\right)\right)$. For example, in Example 2.3.9 one can easily verify that the default conclusion $q$ is derivable at stage 1, i.e. $q £$ $\S_{1}\left(\operatorname{Cons}\left(D^{\S_{1}}\right)\right)$ and hence $q £ C C_{1}$, but not anymore at stage 2 , i.e. $q \quad \S_{2}\left(\operatorname{Cons}\left(D^{\S}\right)\right)$. Note that although $q$ is not derivable as default conclusion at stage 2 this formula is still contained in

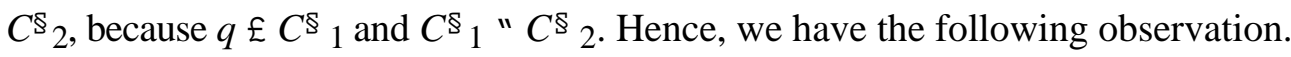

\section{Observation 2.3.15}

If $C^{\S}$ is a constructive extension for the default theory $\Delta=\langle W, D\rangle$, then it can happen that for certain $i$ and $j$ with $i<j$, holds $\S_{\mathrm{i}}\left(\operatorname{Cons}\left(D^{\S_{\mathrm{i}}}\right)\right) \quad \S_{\mathrm{j}}\left(\operatorname{Cons}\left(D^{\S} \mathrm{j}\right)\right)$.

Notice that this non-monotonic increase can be caused either by the constructive character of $C \S$ or the selection function $\$$. In Example 2.3.9 this lack of monotonic increase in the derivable default conclusions happens while the selection function is an identity function. Hence, in this case this effect is not caused by the selection function, but purely by the constructive character of the extension. But this non-monotonic increase can also be caused purely by the selection function: e.g. if $\S_{j}$ makes an empty selection, whereas $\S_{i}\left(\operatorname{Cons}\left(D^{\S_{i}}\right)\right)$ is non-empty.

\section{Proposition 2.3 .16}

If $C \S$ is a constructive extension for the default theory $\Delta=\langle W, D\rangle$, then for each stage $i \geq 1$ holds

$$
C \S_{\mathrm{i}} \text { " } T h\left(W \mathrm{fi}_{\mathrm{j}=0}^{\mathrm{i}} \S_{\mathrm{j}}\left(\operatorname{Cons}\left(D^{\S_{\mathrm{j}}}\right)\right)\right)
$$

where $D^{\S_{\mathrm{i}}}=\left\{\left(:{ }_{1}, \ldots,{ }_{\mathrm{n}} / \bullet\right) £ D \mid \quad £ C_{\mathrm{i}-1}^{\S_{1}}\right.$, and $\left.\quad{ }_{1}, \ldots, \quad{ }_{\mathrm{n}} \quad T h\left(C_{1-1}^{\S_{1}}\right)\right\}$. 


\section{Proof.}

The proof is by induction on the stage $i$ in the construction of the constructive extension $C^{\S}$ for $\Delta=\langle W, D\rangle$.

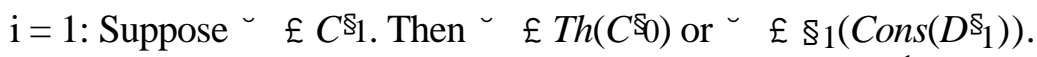

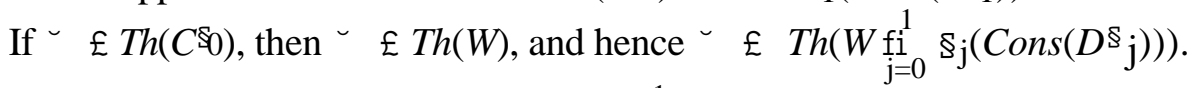

If ${ }^{\sim} \S_{1}\left(\operatorname{Cons}\left(D_{1}^{\$_{1}}\right)\right)$, then $\smile £ \operatorname{Th}\left(W \underset{\mathrm{j}=0}{1} \$_{\mathrm{j}}\left(\operatorname{Cons}\left(D_{\mathrm{j}}\right)\right)\right)$, because

$$
\S_{1}\left(\operatorname{Cons}\left(D^{\S_{1}}\right)\right) "{ }_{\mathrm{j}=0}^{1} \S_{\mathrm{j}}\left(\operatorname{Cons}\left(D^{\S_{\mathrm{j}}}\right)\right)
$$

$\mathrm{i}=\mathrm{m}:$ Suppose $^{\smile} £ C \S_{\mathrm{m}}$, then ${ }^{`} £ \operatorname{Th}\left(C \S_{\mathrm{m}-1}\right)$ or $^{`} £ \S_{\mathrm{m}}\left(\operatorname{Cons}\left(D_{\mathrm{m}}\right)\right)$.

If $\_\operatorname{Th}\left(C \S_{\mathrm{m}-1}\right)$, then $C^{\S_{\mathrm{m}}-1} f$ . Hence, with the induction hypothesis it also follows that $W$ fi ${ }_{\mathrm{j}=0}^{\mathrm{m}=1} \S_{\mathrm{j}}\left(\operatorname{Cons}\left(D^{\S_{\mathrm{j}}}\right)\right) f \quad{ }^{-}$. Since ${ }_{\mathrm{j}=0}^{\mathrm{m}-1} \S_{\mathrm{j}}\left(\operatorname{Cons}\left(D^{\S_{\mathrm{j}}}\right)\right){ }^{\mathrm{m}=0}{ }_{\mathrm{j}=0}^{\mathrm{m}} \S_{\mathrm{j}}\left(\operatorname{Cons}\left(D^{\S_{\mathrm{j}}}\right)\right)$ it also follows that $W$ fi ${ }_{\mathrm{j}=0}^{\mathrm{m}} \S_{\mathrm{j}}\left(\operatorname{Cons}\left(D^{\S_{\mathrm{j}}}\right)\right) f \smile$. Hence, it follows that ${ }^{\smile} \in \operatorname{Th}\left(W \underset{\mathrm{j}=0}{\mathrm{~m}} \S_{\mathrm{j}}\left(\operatorname{Cons}\left(D^{\S_{\mathrm{j}}}\right)\right)\right)$.

If $\smile £ \S_{\mathrm{m}}\left(\operatorname{Cons}\left(D \S_{\mathrm{m}}\right)\right)$, then ${ }^{\smile} £ \operatorname{Th}\left(W \underset{\mathrm{j}=0}{\mathrm{~m}} \S_{\mathrm{j}}\left(\operatorname{Cons}\left(D_{\mathrm{j}}\right)\right)\right)$, because

$$
\S_{\mathrm{m}}\left(\operatorname{Cons}\left(D_{\mathrm{m}}^{\S_{\mathrm{m}}}\right)\right){ }_{\mathrm{j}=0}^{\mathrm{m}} \S_{\mathrm{j}}\left(\operatorname{Cons}\left(D_{\mathrm{j}}\right)\right) . \quad \mathrm{fl}
$$

The converse inclusion of this proposition, i.e. $\operatorname{Th}\left(W \mathrm{fi} \underset{\mathrm{j}=0}{\mathrm{i}} \S_{\mathrm{j}}\left(\operatorname{Cons}\left(D^{\S_{\mathrm{j}}}\right)\right)\right)$ " $C \Im_{\mathrm{i}}$, does not hold. This can be argued as follows. Suppose ${ }^{-} \in \operatorname{Th}\left(W\right.$ fi $\S_{\mathrm{i}}\left(\operatorname{Cons}\left(D_{\uparrow}^{\S}\right)\right)$, then $W$ fi

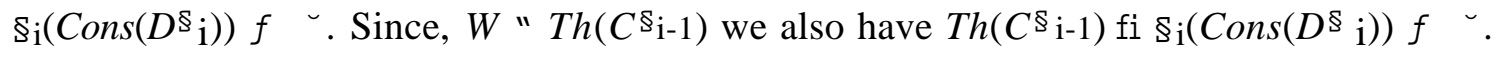
However, this does not yet imply that ${ }^{`} £ \operatorname{Th}\left(C \S_{\mathrm{i}-1}\right)$ fi $\S_{\mathrm{i}}\left(\operatorname{Cons}\left(D^{\S} \mathrm{i}\right)\right)$.

\section{Proposition 2.3.17}

If $C \S$ is a constructive extension for the default theory $\Delta=\langle W, D\rangle$, then

$$
C^{\S}=\operatorname{Th}\left(W \mathrm{fi}_{\mathrm{i}=0} \$_{\mathrm{i}}\left(\operatorname{Cons}\left(D^{\$_{\mathrm{i}}}\right)\right)\right),
$$

where $D^{\S_{\mathrm{i}}}=\left\{\left(:{ }_{1}, \ldots,{ }_{\mathrm{n}} / \bullet\right) £ D \mid £ C_{\mathrm{i}-1}^{\mathbb{1}_{1}}\right.$, and $\left.\quad{ }_{1}, \ldots, \quad{ }_{\mathrm{n}} \quad \operatorname{Th}\left(C_{\mathrm{1}-1}^{\mathbb{S}_{1}}\right)\right\}$.

\section{Proof.}

" : Follows immediately from Proposition 2.3.16, and the fact that $C^{\S} 0=W$.

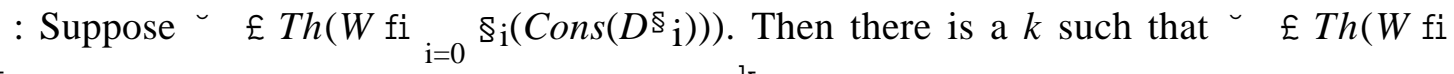

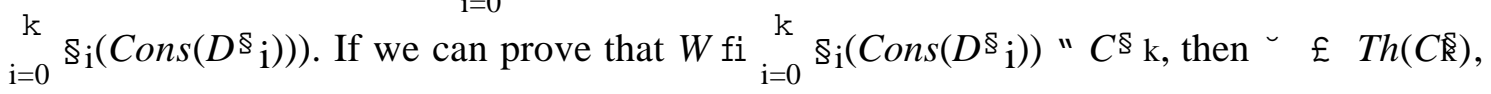
and hence ${ }^{\smile} £ C$ So, we only have to prove that 


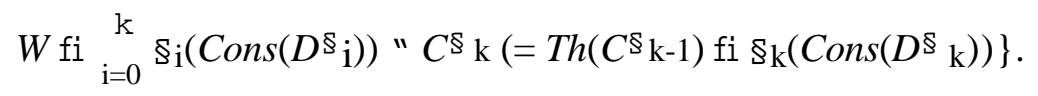

If $\cdot £ W$, then $\cdot £ T h(C \& 1)$, since $W " T h\left(C C_{\mathrm{k}-1}\right)$, and therefore $\cdot £ C{ }_{\mathrm{k}}$.

If $\cdot{ }_{\mathrm{i}=0}^{\mathrm{k}} \S_{\mathrm{i}}\left(\operatorname{Cons}\left(D^{\S_{\mathrm{i}}}\right)\right)$, then there is a $j$ with $0 \leq j \leq k$ such that $\cdot £ \S_{\mathrm{j}}\left(\operatorname{Cons}\left(D^{\S_{\mathrm{j}}}\right)\right)$. This implies that $\cdot £ C \varsigma_{\mathrm{j}}$, and hence also that $\cdot £ C \S_{\mathrm{k}} . \quad \mathrm{fl}$

Satisfying the NSD-constraint is a necessary but not sufficient condition for a selection function to determine an R-extension. It is easy to give examples of selection functions that are NSD, but still do not define an R-extension, simple because they are not exhaustive. For instance some applicable defaults may be not selected at any stage. The notion of exhaustiveness is related to the concept of closure under a set of defaults.

\section{Definition 2.3.18}

Let $D$ be a set of defaults and $S$ be a set of sentences. We call $S$ closed under $D$ if, for every default $\left(: " 1, \ldots,{ }^{n} / \bullet\right) £ D$ with $£ S$ and $\quad{ }_{1}, \ldots, \quad{ }_{n} \quad S, \bullet £ S$. Let $\Delta=<W$, $D>$ be a default theory and suppose $\S$ is a selection function related to $\Delta$. We call $\S$ exhaustive for $D$ if $C^{\S}$ is closed under $D$.

In [TT92] we proved the following theorem which says that constructive extensions which are generated by selection functions that are non-self-defeating and exhaustive are equivalent to Reiter's R-extensions. In other words, the set of all Reiter-extensions of a default theory $\Delta$ is equivalent to the set of all those constructive extensions $C^{\S}$ of $\Delta$ that are generated by non-selfdefeating and exhaustive selection functions that are related to $\Delta$.

\section{Theorem 2.3.19}

Let $\Delta=\langle W, D\rangle$ be a default theory.

$$
\{E \mid E \text { is an R-extension of } \Delta\}
$$$$
=
$$

$\left\{C^{\S} \mid C^{\S}\right.$ is a $\$$-constructive extension of $\Delta$ with $\S$ NSD and exhaustive $\}$

This theorem shows that Reiter's default logic is a special case of constructive default logic.

An interesting class of selection functions is the class of selection fur defeating but not exhaustive. These non-exhaustive $\$$-constructive extensions are respects more intuitive than R-extensions. Lukaszewicz observed in [Luk84, 88] that Rextensions sometimes yield counter-intuitive results. This can be illustrated with the following example. 


\section{Example 2.3.20}

Let $\Delta$ be the default theory with

$$
W={ }^{\circ} \text { and } D=\{(: p / q),(: q / s)\}
$$

The support for the conclusion $q$ is as weak as the support for the conclusion $s$. In the first case the formula $p$ should not be contained in the extension, and in the second case the formula $q$. Since $W$ is empty, one intuitively expects that $\Delta$ has two extensions; one that contains $q$, and another one that contains $s$ (or one extension that contains both conclusions). However, $\Delta$ has only one R-extension, namely $E=T h(\{q\})$, which lacks s. The application of the first default $(: p / q)$ blocks the application of the second default $(: q / s)$, because the justification of the second default is violated by the conclusion of the first one.

With $\$$-constructive extensions these counter-intuitive results can be avoided. Consider two selection functions $\S$ and $\S^{\prime}$, of which the first one never selects the formula $q$, whereas the second is the identity function that selects everything i.e. $\S^{\prime}(V)=V$ for all $V$. It is clear that $C \$$ $=T h(\{s\})$, because the application of $(: q / s)$ is no longer blocked by $(: p / q)$, because the conclusion $q$ is not selected by $\$$. In addition there is also a $\$$-constructive extension of $\Delta, C^{\Im^{\prime}}=E=T h(\{q\})$, which contains the conclusion $q$. In this respect $\$-$ constructive extensions are clearly more intuitive than R-extensions.

In the next sections we will see that the solution of Example 2.3.20 is directly related to the so-called semi-monotonicity property of constructive default logic. A property that Reiter's default logic lacks. Semi-monotonicity is an important property, because it was shown by Guerreiro, Cassanova and Hemerly in [GCH90] that if a default logic is not semimonotonous, then it can never have a proof theory. Reiter observed already in [Rei80] that his default logic does not have, in general, a proof theory, which he considered a serious drawback of his logic.

Constructive extensions have a computational advantage over R-extensions. Selection functions can be used as heuristics to generate extensions more adequately. A more detailed discussion about the computational advantages of constructive default logic in comparison to Reiter's default logic can be found in [TT92]. Of course not all computational problems of default logic that were pointed out so ingeniously by Kautz and Selman in [KS91] are solved by this constructive perspective on R-extensions. In particular the NSD and exhaustive selection functions of \$-constructive extensions that generate R-extensions in Theorem 2.3.19 might be very hard to specify. This specification problem is related to the fact that many of these selection functions, that generate R-extensions, may contain a hidden belief revision component. The NSD-constraint seems to presuppose a kind of look-ahead oracle. A default rule $\partial$, which is applicable at stage $i$, can only be selected at $i$, if its justifications will not be 
violated at any later stage $j>i$. Hence, to determine the selection $\S_{\mathrm{i}}$ at stage $\mathrm{i}$, a look-ahead oracle has to give the information whether justifications will be violated at a later stage $j$. However, this problem of the look-ahead oracle could perhaps be avoided by postponing the checking. Instead of checking whether the justifications of a selected default conclusion will not be violated at a later stage, we simply select those applicable defaults we want, and check at each later stage $j$ whether the then applicable defaults do not have a conclusion that might lead to the violation of an earlier selected default. Hence, this only includes a look-back check on information already known! It is still an open question whether this look-back check works for every NSD selection function. This topic will be further investigated in subsequent research. However, even if no perfect control knowledge can be found, heuristic knowledge may be available that restricts the search and the amount of backtracking needed.

\section{Properties of $\$$-Constructive Extensions}

In this section we will show that constructive default has two important properties that Reiter's default logic lacks. The first property is that every default theory has a constructive extension, and the second property is semi-monotonicity. Both properties are necessary for a proof theory for default logic.

It is well-known that some default theories do not have an R-extension in Reiter's default logic. Consider the following example.

\section{Example 3.1}

Let $\Delta$ be the default theory with $A$ a consistent set of sentences

$$
W=A \text { and } D=\{(: p / p)\}
$$

This default theory has no R-extension $E$.

This lack of extensions (sometimes!) is always considered to be a serious flaw of Reiter's default logic. The problem in Example 3.1 is not so much that the default ( $: p / p$ ) has no $\mathrm{R}$-extension, since this default rule refutes itself. Rather the problem is that the consistent set $A$ has no R-extension at all. Roughly speaking, extensions in default logic can be considered as the conclusion sets of a default theory $\Delta$. Hence, if $\Delta$ has no R-extension, then even the consistent set $A$ has no conclusions in Reiter's default logic. Clearly, this is undesirable. One expects the consistent set $A$ to have at least one extension that contains the deductive closure of $A$, and nothing more. The underlying problem here is that this self-refuting default rule should be ignored in the generation of the extension. Selection functions are a very natural way to obtain this effect. In the general case we can prove that every default theory has at least one $\$$ constructive extension. 


\section{Proposition 3.2 (Existence of $C \$$ )}

Every default theory $\Delta$ has a $\$$-constructive extension $C \S$.

\section{Proof.}

One can easily check that for every default theory $\Delta=\langle W, D\rangle$ the empty selection function $\S$ related to $\Delta$ generates the set $C^{\S}=T h(W)$ which is a constructive extension for $\Delta$.

Since it was already observed that empty selection functions always satisfy the NSDconstraint, it follows immediately from this proof that the existence of constructive extensions is preserved under the NSD-constraint.

\section{Corollary 3.3}

Every default theory $\Delta$ has a $\$$-constructive extension $C \S$ of which the selection function satisfies the NSD-constraint.

Another property which is relevant for default logics is semi-monotonicity. Roughly speaking, semi-monotonicity means that no conclusions are lost when new default rules are introduced.

\section{Definition 3.4 (Semi-Monotonicity)}

Let $\Delta=\langle W, D\rangle$ and $\Delta^{\prime}=\left\langle W, D^{\prime}\right\rangle$ be two default theories such that $D^{\prime}$ " $D$. For any extension $E^{\prime}$ for $\Delta^{\prime}$ there is an extension $E$ for $\Delta$ such that $E^{\prime}$ " $E$.

Reiter's default logic is not semi-monotonous. Consider the following example.

\section{Example 3.5}

Let $\Delta$ and $\Delta^{\prime}$ be two default theories such that

$$
\begin{aligned}
& \Delta: W={ }^{\circ} \text { and } D=\{(: p / q),(: q / s)\} \\
& \Delta^{\prime}: \quad W={ }^{\circ} \text { and } D^{\prime}=\{(: q / s)\}
\end{aligned}
$$

Both default theories have exactly one R-extension. Let $E$ be the R-extension of $\Delta$, and $E^{\prime}$ the R-extension of $\Delta^{\prime}$. Now it is obvious that the formula $s$ is contained in $E$, but not in $E^{\prime}$. Hence, we have $D^{\prime}$ " $D$ and $E^{\prime} \quad E$, which implies that Reiter's default logic is not semimonotonous.

Due to the selection functions we can show that constructive default logic is semimonotonous. 


\section{Proposition 3.6 (Semi-monotonicity of CDL)}

Let $\Delta=\langle W, D\rangle$ and $\Delta^{\prime}=\left\langle W, D^{\prime}\right\rangle$ be two default theories such that $D^{\prime}$ " $D$. For any constructive extension $C^{\S^{\prime}}$ for $\Delta^{\prime}$ there is a selection function $\S$ related to $\Delta$ such that $C^{\S}$ is a constructive extension for $\Delta$ such that $C \S^{\prime}$ " $C$ s.

\section{Proof}

Let $C \S^{\prime}$ be a constructive extension for $\Delta^{\prime}$ generated by the selection function $\S^{\prime}$. The idea of the proof is to define a selection function $\$$ related to $\Delta$ that makes exactly the same selections as $\S^{\prime}$, i.e. $\$$ ignores the conclusions of the extra defaults in $D-D^{\prime}$. Let $D^{\S} \mathrm{i}$ be the set of defaults that are applicable at stage $i$ in the construction of $C^{\S}$, i.e.

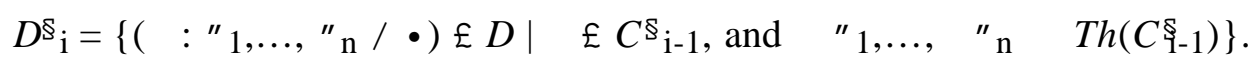

Now we define $\S$ as follows. For all $V$ " $\operatorname{Cons}(D)$, and for all i;

$$
\S_{\mathrm{i}}\left(\operatorname{Cons}\left(D_{\mathrm{i}}\right)\right)=\S_{\mathrm{i}}^{\prime}\left(\operatorname{Cons}\left(D^{\S} \mathrm{i}\right)\right) \text {, and } \S_{\mathrm{i}}(V)={ }^{\circ} \text { if } V \neq \operatorname{Cons}\left(D^{\S} \mathrm{i}\right) \text {. }
$$

Since $\S^{\prime}$ is a function, it is obvious that $\$$ is also a function. Moreover, it also follows immediately from this definition that we have for all $\mathrm{i}$

$$
C{ }^{\lessgtr}=C^{\S} \mathrm{i}
$$

Hence, we have $C \S^{\prime} \mathrm{i}$ " $C{ }^{\S} \mathrm{i}$ for all $\mathrm{i}$. If we can prove that $\S$ is a selection function related to $\Delta$, then it follows immediately from the definition of $\S$ that $C \$$ is a constructive extension of $\Delta$. Since $\S^{\prime}$ is a selection function we know that for all $i$ holds $\S^{\prime}\left(\operatorname{Cons}\left(D^{\S} \mathrm{i}\right)\right)$ " $\operatorname{Cons}\left(D^{\S} \mathrm{i}\right)$. So, if we can prove that for all $i$ holds $\operatorname{Cons}\left(D^{\S} \mathrm{i}\right)$ " $\operatorname{Cons}\left(D^{\S} \mathrm{i}\right)$, then $\$$ is a selection function. Suppose there is a stage $i$ such that $\operatorname{Cons}\left(D^{\S} \mathrm{i}\right) \operatorname{Cons}\left(D^{\S} \mathrm{i}\right)$, then there is a default

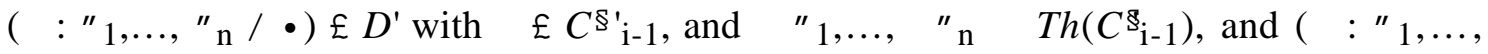

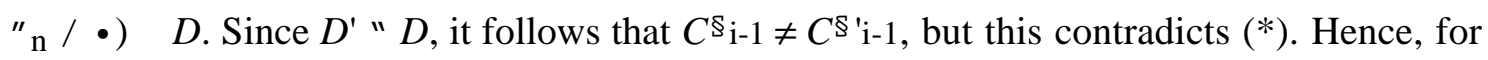
all $i$ holds $\operatorname{Cons}\left(D^{\S} \mathrm{i}\right)$ " $\operatorname{Cons}\left(D^{\S} \mathrm{i}\right)$. fl

\section{A Proof Theory for Constructive Default Logic}

Let $\operatorname{Pre}(D)$ denote the set of prerequisites of the default rules in $D$, and $\operatorname{Jus}(D)$ the set of justifications of default rules in $D$. A proof theory for default logic is defined as follows. We use the definition of Guerreiro, Cassanova and Hemerly from [GCH90], which is a generalization for arbitrary defaults of Reiter's definition of a default proof for normal default as given in [Rei80]. 


\section{Definition 4.1}

Let $\Delta=\langle W, D\rangle$ be a default theory and ${ }^{-}$a sentence. A finite sequence $\left\langle D_{0}, \ldots, D_{\mathrm{k}}\right\rangle$ of finite subsets of $D$ is a default proof of ${ }^{\sim}$ from $\Delta$ iff:

1. $W$ fi $\operatorname{Cons}\left(D_{\mathrm{k}}\right) f^{\smile}$,

2. For $1 \leq i \leq \mathrm{k}, W$ fi $\operatorname{Cons}\left(D_{\mathrm{i}-1}\right) f \operatorname{Pre}\left(D_{\mathrm{i}}\right)$,

3. $D_{0}={ }^{\circ}$,

4. If $W$ is consistent, then for each " $£ \underset{\mathrm{i}=0}{\mathrm{k}} \operatorname{Jus}\left(D_{\mathrm{i}}\right)$ holds

$W$ fi ${ }_{\mathrm{i}=0}^{\mathrm{k}} \operatorname{Cons}\left(D_{\mathrm{i}}\right)$ fi $\{"\}$ is consistent.

The consistency requirement in condition (4) is needed for the following reason. If $W$ is inconsistent, then any finite sequence $\left\langle D_{0}, \ldots, D_{\mathrm{k}}\right\rangle$ of finite subsets of $D$ should be a proof of any sentence ${ }^{-}$from $\Delta$. This property is important in proving that default proof theory is complete.

First we give the definition for soundness and completeness of a default proof theory in the general case, as they are given in [GCH90]. In these definitions $E$ refers to the notion of extension as it is defined in the particular default logic. For example, $E$ refers to R-extensions in Reiter's default logic, and to constructive extensions in constructive default logic.

\section{Definition 4.2 (Soundness of default proof theory)}

If $\left\langle D_{0}, \ldots, D_{\mathrm{k}}\right\rangle$ is a default proof of ${ }^{-}$from $\Delta$, then there is an extension $E$ of $\Delta$ such that ${ }^{-}$ $£ E$.

\section{Definition 4.3 (Completeness of default proof theory)}

If there is an extension $E$ of $\Delta$ such that ${ }^{\checkmark} £ E$, then there is a $\left\langle D_{0}, \ldots, D_{\mathrm{k}}\right\rangle$ which is a default proof of ${ }^{\sim}$ from $\Delta$.

Reiter observed already in [Rei80] that his default logic only has a sound and complete default proof theory for the normal defaults, but not for non-normal ones. It can easily be illustrated why Reiter's default logic is not sound with respect to the default proof theory as defined in Definition 4.1. Consider again the default theory $\Delta=\langle A,\{(: p / p)\}$ of Example 3.1 that has no R-extension. Let ${ }^{`}$ be a formula that follows classically from $A$, i.e. $A f{ }^{`}$. Now it is obvious that the sequence $\left\langle D_{0}\right\rangle$ with $D_{0}={ }^{\circ}$ is a default proof of ${ }^{-}$from $\Delta$. However, since $\Delta$ does not have an R-extension there is certainly not an R-extension $E$ of $\Delta$ that contains the formula $`$. Another reason that makes the proof theory unsound with respect to Reiter's default logic is related to the lack of semi-monotonicity. Consider the two default theories $\Delta=$ $<^{\circ},\{(: p / q),(: q / s)\}>$ and $\Delta^{\prime}=<^{\circ},\{(: q / s)\}>$ from Example 3.5. Now one can easily check that the sequence $\left\langle D_{0}, D_{1}\right\rangle$ with $D_{0}={ }^{\circ}$ and $D_{1}=$ 
$\{(: q / s)\}$ is a default proof of the formula $s$ from $\Delta$ as well as $\Delta^{\prime}$. However, we argued that $\Delta$ has only one R-extension that does not contain the formula $s$.

Gueirrero, Cassanova and Hemerly showed in [GCH90] that the two properties existence and semi-monotonicity of extensions are necessary conditions for a default proof theory which is sound and complete with respect to a particular default logic. In other words, they showed that if a particular default logic does not have the existence of extensions property or the semimonotonicity property, then it is impossible to define a default proof theory that is sound and complete with respect to this default logic (see Theorem 13 in [GCH90]).

Since we showed in the previous section that constructive default logic has the existence property as well as the semi-monotonicity property, it satisfies the necessary conditions for a sound and complete proof theory. However, necessary conditions are not yet sufficient conditions. We still have to prove positively that constructive default logic has a default proof theory. This is done in the proofs of the following theorems.

\section{Theorem 4.4 (Soundness of default proof theory w.r.t. CDL)}

If there is a default proof of ${ }^{\sim}$ from $\Delta=\langle W, D\rangle$, then there is a constructive extension $C^{\S}$ of $\Delta$ such that ${ }^{\smile} £ C$.

\section{Proof.}

Suppose $~$ has a default proof from $\Delta=\langle W, D\rangle$. Then there is a finite sequence $<\mathrm{B}_{0}, \ldots, \mathrm{B}_{\mathrm{k}}>$ of finite subsets $\mathrm{B}_{\mathrm{i}}$ of $D$ such that it is a default proof of ${ }^{\sim}$ from $\Delta$. We use this sequence to generate a constructive extension $C^{\S}$ of $\Delta$ that contains the formula $~$ in the following way.

Let $D^{\$_{i}}$ be again the set of defaults that are derivable at stage $i$ in the construction of $C$, i.e. for $i>1$ we define

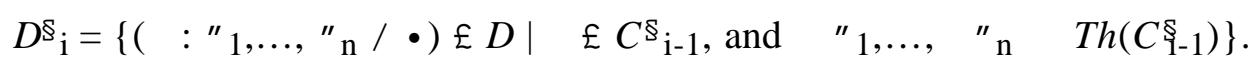

Now define the selection function $\S$ that is used to generate $C^{\S}$ as follows;

$$
\begin{aligned}
& \S_{2 \mathrm{n}}\left(\operatorname{Cons}\left(D^{\S_{2}}\right)\right)=\operatorname{Cons}\left(\mathrm{B}_{\mathrm{n}}\right), \text { for } 0 \leq n \leq \mathrm{k}, \\
& \S_{2 \mathrm{n}+1}\left(\operatorname{Cons}\left(D^{\S_{2 n+1}}\right)\right)={ }^{\circ}, \text { for } 0 \leq n \leq \mathrm{k}, \\
& \S_{\mathrm{n}}\left(\operatorname{Cons}\left(D^{\S_{2}+1}\right)\right)={ }^{\circ}, \text { for } n>2 \mathrm{k},
\end{aligned}
$$$$
\text { and for any } V \text { and any } i \text { such that } V \neq \operatorname{Cons}\left(D^{\S_{\mathrm{i}}}\right) \text {, we have } \S_{\mathrm{i}}(V)={ }^{\circ} \text {. }
$$ 
If we can prove that $\$$ is a selection function, then it follows immediately from the definition of $\$$ that $C \$$ is a constructive extension for $\Delta$ such that ${ }^{-} £ C$ $C^{\$}$. So, what remains to be proven is that this $\$$ is a selection function.

Due to the definition given above it is trivially true that $\$$ is a function. To prove that $\$$ also satisfies the requirement of a selection function we have to prove that for all stages $i$ in the construction of $C \S$ holds $\S_{\mathrm{i}}\left(\operatorname{Cons}\left(D_{1}^{\$_{1}}\right)\right)$ " $\operatorname{Cons}\left(D^{\S_{\mathrm{i}}}\right)$. Note that this trivially holds for every stage $i$ with an empty selection; i.e. for $i=2 \mathrm{n}+1$ with $1 \leq n \leq k$, and for $i>2 k$. Hence, we only have to prove

$$
\operatorname{Cons}\left(\mathrm{B}_{\mathrm{i}}\right) " \operatorname{Cons}\left(D^{\S} 2 \mathrm{i}\right) \text {, for } 0 \leq i \leq k .
$$

Note that by definition $\mathrm{B}_{0}={ }^{\circ}$. Suppose $-£ \operatorname{Cons}\left(\mathrm{B}_{\mathrm{i}}\right)$ with $1 \leq \mathrm{i} \leq \mathrm{k}$, then there is a default $\partial$ $=\left(:{ }^{\prime} 1, \ldots,{ }_{n} / \bullet\right) £ \mathrm{~B}_{\mathrm{i}}$, and therefore we also have

$$
\left(:{ }_{1}, \ldots,{ }_{\mathrm{n}} / \bullet\right) £ D .
$$

Since, $<\mathrm{B}_{0}, \ldots, \mathrm{B}_{\mathrm{k}}>$ is a default proof it follows that for any $i$ with $1 \leq i \leq k$ holds $W$ fi $\operatorname{Cons}\left(\mathrm{B}_{\mathrm{i}-1}\right) f \operatorname{Pre}\left(\mathrm{B}_{\mathrm{i}}\right)$, and consequently it follows in particular that $W$ fi $\operatorname{Cons}\left(\mathrm{B}_{\mathrm{i}-1}\right) f$. Hence, by definition of $\$$ it also follows that $W$ fi $\S_{2(i-1)}\left(\operatorname{Cons}\left(D^{\S} 2(\mathrm{i}-1)\right)\right) f$, and thus we have $\quad £ T h\left(C S_{2(\mathrm{i}-1)}\right)$, and hence it follows that

$$
£ C^{\$_{\mathrm{i}-1}}
$$

Let $S D^{\S_{i}}$ denote the set of applicable default rules from $D^{\S_{i}}$ of which the conclusions are selected by $\S_{i}$, i.e.

$$
S D^{\$_{i}}=\left\{\left(\quad: " 1, \ldots,{ }_{n} / \bullet\right) £ D_{\mathrm{i}} \mid \cdot £ \S_{\mathrm{i}}\left(\operatorname{Cons}\left(D_{\mathrm{i}}\right)\right)\right\}
$$

By definition of default proof it follows that each " $£ \underset{i=0}{k} \operatorname{Jus}\left(B_{i}\right)$ is consistent with $W$ fi ${ }_{\mathrm{i}=0}^{\mathrm{k}} \operatorname{Cons}\left(\mathrm{B}_{\mathrm{i}}\right)$. Hence, this implies that

$$
\text { each " } £ \underset{\mathrm{i}=0}{2 \mathrm{k}} \operatorname{Jus}\left(S D^{\S_{\mathrm{i}}}\right) \text { is consistent with } W \text { fi }{ }_{\mathrm{i}=0}^{2 \mathrm{k}} \operatorname{Cons}\left(S D^{\S_{\mathrm{i}}}\right) \text {. }
$$

Since we know from Proposition 2.3.17 that $C^{\S}=T h\left(W \mathrm{fi}_{\mathrm{i}=0} \S_{\mathrm{i}}\left(\operatorname{Cons}\left(D^{\S} \mathrm{i}\right)\right)\right.$, we also know that $C^{\S}=T h\left(W \mathrm{fi}_{\mathrm{i}=0}^{2 \mathrm{k}} \S_{\mathrm{i}}\left(\operatorname{Cons}\left(D^{\S}\right)\right)\right.$, because after stage $2 k$ no new default conclusions are selected by $\$$. Since ${ }_{\mathrm{i}=0}^{2 \mathrm{k}} \S_{\mathrm{i}}\left(\operatorname{Cons}\left(D^{\S_{\mathrm{i}}}\right)\right)={ }_{\mathrm{i}=0}^{2 \mathrm{k}} \operatorname{Cons}\left(S D^{\S_{\mathrm{i}}}\right)$, it follows from (3) that for all justifications " $1, \ldots,{ }_{n}$ from the default rule $\partial$ we have $"{ }_{1}, \ldots$, "n $C$, and hence also 


$$
\text { " } 1, \ldots, \quad \text { "n } \operatorname{Th}\left(C \mathcal{S}_{\mathrm{i}-1}\right)
$$

From (1), (2) and (4) it follows that $\partial £ D^{\S} 2 \mathrm{i}$, and therefore its conclusion $\bullet$ is contained in Cons $\left(D^{\S} 2 \mathrm{i}\right)$. Consequently, $\$$ is a selection function.

fl

We can even prove a stronger type of soundness, namely that if there is a default proof of from $\Delta=\langle W, D\rangle$, then there is a constructive extension $C^{\S}$ of $\Delta$ with a selection function $\S$ that is NSD such that ${ }^{-} £ C$. This type of soundness is called NSD-soundness.

\section{Theorem 4.5 (NSD-soundness of default proof theory w.r.t. CDL)}

If there is a default proof of ${ }^{\sim}$ from $\Delta=\langle W, D\rangle$, then there is a constructive extension $C \$$ of $\Delta$ with a selection function $\$$ that is NSD such that ${ }^{-} £ C$.

\section{Proof.}

Suppose $\checkmark$ has a default proof from $\Delta=\langle W, D\rangle$, then there is a finite sequence $<\mathrm{B}_{0}, \ldots, \mathrm{B}_{\mathrm{k}}>$ of finite subsets of $D$ such that it is a default proof of ${ }^{-}$from $\Delta$. We use this sequence to generate a constructive extension $C^{\S}$ of $\Delta$ that contains the formula ${ }^{-}$by defining the selection function $\S$ exactly as in the proof of Theorem 4.4. We prove that $\$$ is NSD by contraposition.

Suppose that $\$$ is not NSD, i.e. $\$$ is self-defeating. Hence, there is a stage $i$ and a formula $\bullet$ that is selected by $\$$ at stage $i$ such that for every applicable default rule $\left(: " 1, \ldots,{ }^{n} / \bullet\right) £$ $D \S_{\mathrm{i}}$ with conclusion • there is some later stage $j>i$ such that ${ }^{\prime}{ }_{\mathrm{m}} £ \operatorname{Th}\left(C \S_{\mathrm{j}}\right)$ with $1 \leq m \leq n$. This implies that " $\mathrm{m} £ C$, hence by Proposition 2.3.17 it also follows that $"{ }_{\mathrm{m}} £ \operatorname{Th}\left(W \mathrm{fi}_{\mathrm{i}=0} \S_{\mathrm{i}}\left(\operatorname{Cons}\left(D_{\mathrm{i}}\right)\right)\right)$. Since every selection of $\S$ after stage $2 k$ is by definition empty, it follows that

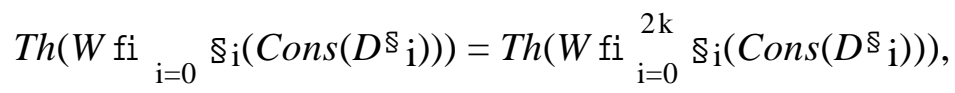

and hence $\quad{ }^{\mathrm{m}} £ \operatorname{Th}\left(W \mathrm{fi}_{\mathrm{i}=0}^{2 \mathrm{k}} \S_{\mathrm{i}}\left(\operatorname{Cons}\left(D^{\S_{\mathrm{i}}}\right)\right)\right)$. Since,

$$
{ }_{\mathrm{i}=0}^{2 \mathrm{k}} \S_{\mathrm{i}}\left(\operatorname{Cons}\left(D^{\S_{\mathrm{i}}}\right)\right)={ }_{\mathrm{i}=0}^{\mathrm{k}} \operatorname{Cons}\left(\mathrm{B}_{\mathrm{i}}\right),
$$

it follows that $W$ fi ${ }_{\mathrm{i}=0}^{\mathrm{k}} \operatorname{Cons}\left(\mathrm{B}_{\mathrm{i}}\right)$ fi $\left\{{ }^{\prime \prime} \mathrm{m}\right\}$ is inconsistent, which contradicts the assumption that the sequence $<\mathrm{B}_{0}, \ldots, \mathrm{B}_{\mathrm{k}}>$ satisfies condition (4) of the definition of a default proof. $\mathrm{fl}$

We can only prove completeness with respect to a subclass of constructive extensions; namely the extensions that are NSD. This restriction is needed for the following reason. Consider again Example 2.3.9. In this example there was a default theory $\Delta$ with $W=\left\{\begin{array}{ll}q & p\end{array}\right\}$ and $D=\{(: p / q)\}$. This $\Delta$ has a constructive extension $C^{\S}=T h(\{q, p\})$ of which the 
selection function $\$$ is not NSD. One can easily verify that the only way to derive the formula $q$ with a default proof from $\Delta$ is with a sequence $\left\langle\mathrm{B}_{0}, \ldots, \mathrm{B}_{\mathrm{k}}\right\rangle$ such that $(: p / q) £ \mathrm{~B}_{\mathrm{k}}$. However, with respect to this sequence we have that $W$ fi $\underset{\mathrm{i}=0}{\mathrm{k}} \operatorname{Cons}\left(\mathrm{B}_{\mathrm{i}}\right)$ fi $\{p\}$ is inconsistent, hence it violates condition (4) of the definition of a default proof, and therefore this sequence is not a default proof. This argument can be generalized to a proof for the following theorem.

\section{Theorem 4.6}

If $C \S$ is a constructive extension for a default theory $\Delta=\langle W, D\rangle$ with a selection function $\S$ that is not NSD, then $C^{\S}$ contains at least one formula ${ }^{\sim}$ for which there exists no default proof from $\Delta$.

Proof. Straightforward.

\section{Theorem 4.7 (NSD-completeness of default proof theory w.r.t. CDL)}

Let $C \S$ be a constructive extension of a default theory $\Delta=\langle W, D\rangle$ where $D$ is a finite set of defaults. If a formula ${ }^{-}$is contained in $C \S$ and the selection function $\$$ is NSD, then there is a default proof of ${ }^{\sim}$ from $\Delta$.

\section{Proof.}

Let the formula ${ }^{\sim}$ be contained in a constructive extension $C^{\S}$ of a default theory $\Delta=$ $<W, D>$ with a selection function $\S$ that is NSD. Let $D^{\S_{i}}$ be the set of defaults that are derivable at stage $i$ in the construction of $C \S$, i.e. for $i>0$ we define again

$$
D_{\mathrm{i}}^{\S_{i}}=\left\{\left(:{ }_{1}, \ldots,{ }_{\mathrm{n}} / \bullet\right) £ D \mid £ C_{\mathrm{i}-1}^{\S_{\mathrm{i}}} \text {, and } \quad{ }_{1}, \ldots, \quad{ }_{\mathrm{n}} \quad \operatorname{Th}\left(C_{1-1}^{\S_{1}}\right)\right\}
$$

And let $S D^{\S_{i}}$ again denote the set of applicable default from $D^{\S_{i}}$ of which the conclusions are selected by $\S_{i}$, i.e.

$$
S D^{\S_{i}}=\left\{\left(\quad:{ }_{1}, \ldots,{ }_{n} / \bullet\right) £ D \Im_{\mathrm{i}} \mid \cdot £ \S_{\mathrm{i}}\left(\operatorname{Cons}\left(D_{\mathrm{i}}\right)\right)\right\}
$$

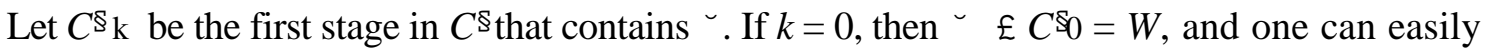
verify that the sequence $\left\langle\mathrm{B}_{0}\right\rangle$ with $\mathrm{B}_{0}={ }^{\circ}$ is a default proof of ${ }^{-}$from $\Delta$. If $k>0$, then define each set $B_{i}$ in the finite sequence $\left\langle B_{0}, \ldots, B_{k}>\right.$ as follows

$$
\mathrm{B}_{0}={ }^{\circ} \text { and } \mathrm{B}_{\mathrm{i}}={ }_{\mathrm{j}=0}^{\mathrm{i}} S D^{\S_{\mathrm{j}}} \text {, for } 1 \leq i \leq \mathrm{k} \text {. }
$$

It is obvious that $\left\langle\mathrm{B}_{0}, \ldots, \mathrm{B}_{\mathrm{k}}\right\rangle$ is a finite sequence of finite subsets, because the set of defaults $D$ is finite. We have to prove that this sequence is a default proof. We check the four conditions of Definition 4.1. 


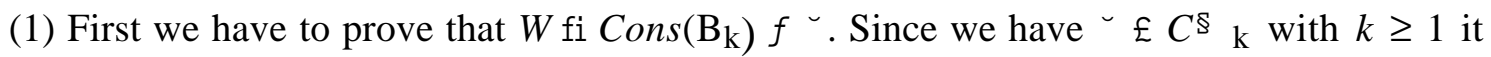
follows by Proposition 2.3.16 that ${ }^{\smile} £ T h\left(W \mathrm{fi}_{\mathrm{j}=0}^{\mathrm{k}} \S_{\mathrm{j}}\left(\operatorname{Cons}\left(D^{\S_{\mathrm{j}}}\right)\right)\right)$. Since

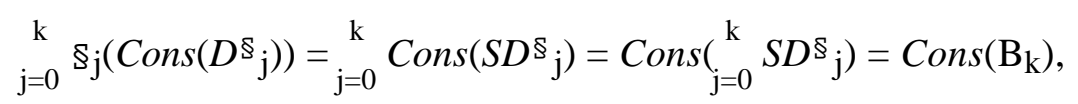

it follows that ${ }^{\smile} £ \operatorname{Th}\left(W\right.$ fi $\left.\operatorname{Cons}\left(\mathrm{B}_{\mathrm{k}}\right)\right)$, and hence $W$ fi $\operatorname{Cons}\left(\mathrm{B}_{\mathrm{k}}\right) f^{\smile}$.

(2) Next we have to prove that for each $i$ with $1 \leq i \leq k$ holds

$$
W \text { fi } \operatorname{Cons}\left(\mathrm{B}_{\mathrm{i}-1}\right) f \operatorname{Pre}\left(\mathrm{B}_{\mathrm{i}}\right) \text {. }
$$

Let $£ \operatorname{Pre}\left(\mathrm{B}_{\mathrm{i}}\right)$, then there is a default $\left(:{ }_{1}, \ldots,{ }^{\prime}{ }_{\mathrm{n}} / \bullet\right) £ \mathrm{~B}_{\mathrm{i}}$. Since, $\mathrm{B}_{\mathrm{i}}{ }_{\mathrm{j}=0}^{\mathrm{i}} S D^{\S_{\mathrm{j}}}$ we also know that there is an $m$ with $0 \leq m \leq i$ such that $\left(:{ }^{\prime}{ }_{1}, \ldots,{ }^{n} / \bullet\right) £ S D_{\mathrm{m}}^{\mathbb{\mathrm { m }}_{\mathrm{m}}}$, and hence $£ C{ }^{-} \mathrm{m}-1$.

Consequently, by Proposition 2.3.16 it follows that $£ \quad T h\left(W f i{ }_{j=0}^{m-1} \S_{j}\left(\operatorname{Cons}\left(D^{\S_{j}}\right)\right)\right)$. Since $m \leq i$, it follows that $£ \operatorname{Th}\left(W \mathrm{fi}_{\mathrm{j}=0}^{\mathrm{i}-1} \S_{\mathrm{j}}\left(\operatorname{Cons}\left(D^{\S_{\mathrm{j}}}\right)\right)\right)$. Since,

$$
{ }_{\mathrm{j}=0}^{\mathrm{i}-1} \S_{\mathrm{j}}\left(\operatorname{Cons}\left(D_{\mathrm{j}}^{\S_{\mathrm{j}}}\right)\right)={ }_{\mathrm{j}=0}^{\mathrm{i}-1} \operatorname{Cons}\left(\operatorname{SD}{ }_{\mathrm{j}}\right)=\operatorname{Cons}\left({ }_{\mathrm{j}=0}^{\mathrm{i}-1} \operatorname{SD}_{\mathrm{j}}\right)=\operatorname{Cons}\left(\mathrm{B}_{\mathrm{i}-1}\right),
$$

it also follows that $\quad £ \operatorname{Th}\left(W\right.$ fi $\left.\operatorname{Cons}\left(\mathrm{B}_{\mathrm{i}-1}\right)\right)$, and hence $W$ fi $\operatorname{Cons}\left(\mathrm{B}_{\mathrm{i}-1}\right) f \quad$.

(3) $\mathrm{B}_{0}={ }^{\circ}$ follows by definition.

(4) Finally, we have to show that if $W$ is consistent, then for each " $£ \underset{i=0}{\mathrm{k}} \operatorname{Jus}\left(\mathrm{B}_{\mathrm{i}}\right)$ holds that $W$ fi ${ }_{\mathrm{i}=0}^{\mathrm{k}} \operatorname{Cons}\left(\mathrm{B}_{\mathrm{i}}\right)$ fi $\left\{{ }^{\prime \prime}\right\}$ is consistent. Assume $W$ is consistent, and that for a certain "' $£$ ${ }_{\mathrm{i}=0}^{\mathrm{k}} \operatorname{Jus}\left(\mathrm{B}_{\mathrm{i}}\right)$ the set of sentences $W$ fi $\underset{\mathrm{i}=0}{\mathrm{k}} \operatorname{Cons}\left(\mathrm{B}_{\mathrm{i}}\right) \mathrm{fi}\left\{{ }^{\prime \prime}\right\}$ is inconsistent. Note that ${ }_{\mathrm{i}=0}^{\mathrm{k}} \mathrm{B}_{\mathrm{i}}=$ $\mathrm{B}_{\mathrm{k}}$, hence it follows that

$$
\underset{\mathrm{i}=0}{\mathrm{k}} \operatorname{Jus}\left(\mathrm{B}_{\mathrm{i}}\right)=\operatorname{Jus}\left({ }_{\mathrm{i}=0}^{\mathrm{k}} \mathrm{B}_{\mathrm{i}}\right)=\operatorname{Jus}\left(\mathrm{B}_{\mathrm{k}}\right)
$$

Let $S D^{\S_{\mathrm{j}}}$ be the first stage in $\mathrm{B}_{\mathrm{k}}$ in which a default is applicable with the justification "' of which the conclusion is selected by $\S_{\mathrm{j}}$. Since this default is applicable in $S D^{\$_{\mathrm{j}}}$, it follows that "' $T h\left(C^{\S}{ }_{\mathrm{j}-1}\right)$. But this implies that "' must have been derived at a later stage $C^{\S_{\mathrm{m}}}$ with $j \leq m \leq k$, which contradicts the assumption that the selection function $\$$ is NSD. fl 


\section{Conclusions}

Reiter's original default logic lacks a default proof theory for non-normal default rules, which was already observed by Reiter himself to be a weakness of his logic. In this paper we have shown that constructive default logic does have a default proof theory. We have also shown that this is intrinsically related to the fact that default theories in $C D L$ always have a constructive extension (the existence property), and that $C D L$ also has the semi-monotonicity property. Furthermore, we have shown that, with respect to some counter-examples that were suggested by Lukaszewicz, constructive extensions yield more intuitive conclusions than Reiter's extensions. Hence, constructive default logic does not only have heuristic advantages over default theory from a computational point of view, but it is also more adequate with respect to knowledge representation.

\section{Acknowledgements}

I wish to thank Witold Lukaszewicz for his careful proofreading and many helpful suggestions, and Joeri Engelfriet for his remarks.

\section{References}

[Bes89] P. Besnard, An Introduction to Default Logic, Springer Verlag, 1989.

[Bel77] N. D. Belnap, A useful four-valued logic, in: J.M. Dunn and G. Epstein (eds.), Modern Uses of Multiple-Valued Logic, Reidel, Dordrecht, 1977, pp. 5-37.

[Bla86] S. Blamey, Partial Logic, in: D. Gabbay and F. Guenthner (eds.), Handbook of Philosophical Logic, Vol.III, Reidel, 1986, pp. 1-70.

[Bre89] G. Brewka, Preferred subtheories: An extended logical framework for default reasoning, Proc. of the IJCAI '89, Detroit, 1989, pp. 1043-1048.

[Bre91] G. Brewka, Cumulative Default Logic: in defense of nonmonotonic inference rules, Artificial Intelligence 50, 1991, pp. 183-205.

[dC74] N. da Costa, On the theory of inconsistent formal systems, Notre Dame Journal of Formal Logic XV, 1974, pp.497-510.

[GH91] D. Gabbay and T. Hunter, Making inconsistency respectable, Part I, in: Ph. Jorrand and J. Keleman (eds.), Fundamentals of Artificial Intelligence Research, Lecture Notes in Artificial Intelligence, Vol. 535, Springer Verlag, 1992. 
[GH92] D. Gabbay and T. Hunter, Making inconsistency respectable, Part II, Technical report, Dept. of Computing, Imperial college, London, 1992.

[Eth88] D. W. Etherington, Reasoning with Incomplete Information, Pitman, London, 1988.

[FM92a] C. Froidevaux and J. Mengin, A framework for comparing default logics, In: D. Pearce and G. Wagner (eds.), Proc. of JELIA 92, Vol. 633, Lecture Notes in Artificial Intelligence, Springer Verlag, 1992, pp. 154-173.

[FM92b] C. Froidevaux and J. Mengin, An Operational Approach to Default Logics, Tech. Report, Laboratoire de Recherche et Informatique, Université Paris Sud, 1992.

[GCH90] R.A. Gueirrero, M.A. Cassanova and A.S. Hemerly, Contributions to a proof theory for generic defaults, Proc. ECAI 90, 1990.

[JB91] U. Junker and G. Brewka, Handling partially ordered defaults in TMS, in: R. Kruse and P. Siegel (eds.), Symbolic and Quantitative Approaches to Uncertainty, Lecture Notes in Computer Science 548, Springer Verlag, 1991, pp. 211-218.

[Jun92] U. Junker, Controlling the selection and retraction of assumptions, Proc. of the ECAI'92 Workshop on Theoretical Foundations of Knowledge Representation, Vienna, 1992.

[KS91] H. Kautz and B. Selman, Hard problems for simple default theories, Artifical Intelligence 49, 1991, pp.243-279.

[Luk84] W. Lukaszewicz, Considerations on default logic, Proc. AAAI Workshop on NonMonotonic Reasoning, New Paltz, 1984, pp.165-193.

[Luk88] W. Lukaszewicz, Considerations on default logic - An alternative approach, Computational Intelligence, 4:1-16, 1988.

[Luk90] W. Lukaszewicz, Non-monotonic Reasoning, Series in Artificial Intelligence, Ellis Horwood, 1990.

[Moi92] Y. Moinard, Unifying various approaches to default logic, Proc. of the IPMU '92, Mallorca,1992.

[Pea92] D. Pearce, Default logic based on constructive logic, In: B. Neumann (ed.), Proc. of the European Conference on Artificial Intelligence, ECAI '92, John Wiley and Sons, 1992. 
[Rei80] R. Reiter, A logic for default reasoning, Artificial Intelligence 13, pp. 81-132.

[Tan91] Y.H. Tan, Relating bi-modular systems to default logic and autoepistemic logic, Proc. Int. Workshop on Non-monotonic Reasoning and Partial semantics, 1991, Free University Amsterdam.

[Tan92a] Y.H. Tan, Non-monotonic Reasoning: Logical Architecture and Philosophical Applications, Ph.D. Thesis, Free University Amsterdam, Department of Mathematics and Computer Science, 1992.

[Tan92b] Y.H. Tan, BMS - A meta-level approach to non-monotonic reasoning, in: W. Van der Hoek, J.-J. Ch. Meyer, Y.H. Tan and C. Witteveen (eds.), Non-Monotonic Reasoning and Partial Semantics, Series in Artificial Intelligence, Ellis Horwood Publishers, 1992.

[Tan92c] Y.H. Tan, Representing a meta-level architecture for non-monotonic reasoning in (extended) partial logic, Proc. of the Workshop Partial Semantics and Non-Monotonic Reasoning, University of Linkoping, May 25-29, 1992.

[Tan93] Y.H. Tan, Merging object-level and meta-level logics using two types of negations, Tech. Report, Erasmus University Rotterdam, 1993.

[TT90] Y.H. Tan and J. Treur, Partial logic and non-monotonic reasoning, Proc. ECAI-90 Workshop on Partial Deduction, Partial Evaluation and Intelligent Reasoning, Stockholm, 1990.

[TT91a] Y.H. Tan and J. Treur, A bi-modular approach to non-monotonic reasoning, in: F. De Glas and D. Gabbay (eds.), Proc. World Congress on Fundamentals of Artificial Intelligence, WOCFAI-91, Paris, 1991, pp. 461-476. (An modified version will appear in a special volume of Studia Logica.)

[TT92] Y.H. Tan and J. Treur, Constructive default logic and the control of defeasible reasoning, In: B. Neumann (ed.), Proc. of the European Conference on Artificial Intelligence, ECAI '92, John Wiley and Sons, 1992, pp. 299-303. (Extended version: Report IR-280, Department of Mathematics and Computer Science, Vrije Universiteit Amsterdam, 1991, 52 pp.) 